\title{
Management of a South African family with retinitis pigmentosa - should potential therapy influence translational research protocols?
}

\author{
Lisa Roberts • George Rebello • \\ Rajkumar Ramesar • Jacquie Greenberg
}

Received: 7 December 2007 / Accepted: 13 February 2008/Published online: 29 May 2008

(C) Humana Press 2008

\begin{abstract}
Mutation analysis of retinal candidate genes is performed as part of an ongoing research to identify the causative genetic defect in South African families with retinal degenerative disorders (RDDs). A translational research protocol has been established whereby probands are counseled and given their molecular genetic results to take back to other family members, who can then request individual diagnostic testing. A Thr17Met mutation of the rhodopsin gene was identified in a Caucasian South African family with autosomal dominant retinitis pigmentosa. Patients with this mutation appear to benefit from treatment using oral vitamin A supplementation. This family has been informed that a molecular diagnosis is available; however, one individual has refused testing and none of the younger generation has shown interest in receiving molecular results or genetic counseling. Adapting the established protocol for the translation of RDD research results and contacting mutation positive individuals may be justifiable in light of the potential benefit of therapy.
\end{abstract}

Keywords Retinitis pigmentosa $\cdot$ Rhodopsin $\cdot$ Vitamin A . Translational research $\cdot$ Ethics

L. Roberts $\cdot$ G. Rebello $\cdot$ R. Ramesar $\cdot$ J. Greenberg

UCT/MRC Human Genetics Unit, Division of Human Genetics, IIDMM, Department of Clinical Laboratory Sciences,

University of Cape Town,

Cape Town, South Africa

L. Roberts $(\square)$

Ramesar Laboratory, Institute of Infectious Disease and Molecular Medicine, Faculty of Health Sciences, University of Cape Town, Level 3 Wernher \& Beit North Building, Observatory 7925,

Cape Town, South Africa

e-mail: Lisa.Roberts@uct.ac.za

\section{Introduction}

Retinitis pigmentosa (RP) is a group of diseases characterized by photoreceptor cell death in the retina and subsequent vision loss. Symptoms include night blindness, followed by peripheral vision loss and decreasing visual fields [1]. Total blindness occurs in $30 \%$ of cases [1, 2]. Approximately $65 \%$ of RP is nonsyndromic, and of these cases approximately $30 \%$ show autosomal dominant Mendelian inheritance.

Autosomal dominant retinitis pigmentosa (adRP) can be classified into two main clinical types. In individuals with type 1 adRP, onset of symptoms occurs early (in the first decade of life). In individuals with type 2 adRP, onset of symptoms begins later (in the second or third decade of life) and can vary within or between families, with asymptomatic mutation carriers being reported [3]. There are currently 17 different genes implicated in causing adRP. Mutations in the rhodopsin gene are causative of approximately $27 \%$ of adRP [1].

A research project was initiated at the Division of Human Genetics at the University of Cape Town in 1990 to investigate retinal degenerative disorders (RDD) in South Africa. The goal of this ongoing research is to identify the causative genetic mutation of each family in the RDD DNA database to facilitate clinical and genetic management and future therapeutic intervention. Once a mutation has been identified in a family, the research results are translated into a diagnostic service where diagnostic, predictive, carrier, prenatal, and pre-implantation testing can be offered [4]. The amount of information, which can be given regarding prognosis of disease, potential therapies, and eligibility for treatment trials, however, depends on how well the causative gene and/or mutation has been characterized. 


\section{Materials and methods}

This study has been approved by the Research Ethics Committee of the Faculty of Health Sciences, University of Cape Town (UCT). Informed consent has been obtained from subjects, and the study complies with the Declaration of Helsinki (2000). We certify that all applicable institutional and governmental regulations concerning the ethical use of human volunteers were followed during this research.

Mutation analysis was performed across the entire coding sequence of the rhodopsin gene $(R H O)$ in a cohort of 47 affected, unrelated individuals with adRP. Peripheral blood samples were obtained and genomic DNA was isolated from white blood cells, using standard methods. DNA from each individual was subjected to PCR amplification and single-stranded conformational polymorphism analysis (SSCP), using previously published primers [5]. All samples exhibiting variant SSCP profiles were characterized by direct sequencing using the ABI Prism ${ }^{\mathrm{TM}} 377$ automated sequencer (Applied Biosystems, USA), according to manufacturers instructions.

One pathogenic mutation, c.50C $>\mathrm{T}$ (which encodes a Thr17Met amino acid change) was identified in $R H O$ exon 1 of an affected Caucasian proband. The Thr17Met mutation creates a $B s t \mathrm{X}$ I restriction enzyme site, therefore a restriction enzyme digest protocol was optimized to create an accurate, reliable diagnostic assay. The enzyme digest was performed as follows: $15 \mu \mathrm{l}$ RHO exon 1 PCR product, 1U BstXI (Roche Diagnostics, South Africa) and $1 \times$ SuRE/ Cut Buffer were incubated in a reaction volume of $20 \mu \mathrm{l}$ at $45^{\circ} \mathrm{C}$ for 16 hours. Products of the restriction enzyme digest were separated on a $3 \%$ agarose gel containing ethidium bromide and visualized under ultraviolet light. The enzyme digest was performed on all available samples from relatives to ensure co-segregation of the variant with disease in this family.

The research results were translated into a diagnostic service according to a protocol established by a team, which included genetic counselors, medical scientists, coprincipal investigators, and a clinical coordinator. The established protocol is as follows:

1. Patients (individuals and/or families) are recruited for research, and DNA from the proband is screened for mutations.

2. If a mutation is detected in the proband, DNA from the family is tested for that mutation (at this point the proband will have been retested to confirm the screening result, and the family members will have been tested once).

3. The proband is contacted, counseled, and given the molecular results to take back to the rest of the family.

4. If a family member requests an individual diagnostic result, DNA from that individual will be retested after appropriate counseling, depending on whether the individual is symptomatic or asymptomatic. Ideally two tests are performed on two different DNA samples, isolated on different days. This is assessed on a case by case basis, depending on risk status and pretest counseling.

5. A report is written and the results are delivered, after discussion by the management team.

\section{Results}

A well-characterized rhodopsin mutation, Thr17Met, was identified in a Caucasian South African family. The family pedigree is shown in Fig. 1 and clinical information of
Fig. 1 Pedigree of the South African family with adRP caused by a Thr17Met mutation of rhodopsin. In the pedigree shown, standard symbols are used-squares represent males and circles represent females. Shaded symbols indicate affected individuals, and a symbol with a line through it indicates that the individual is deceased. Individual 63.2 (III: 5) was the proband, and individuals 63.163.5 are individuals from whom blood was taken for research purposes

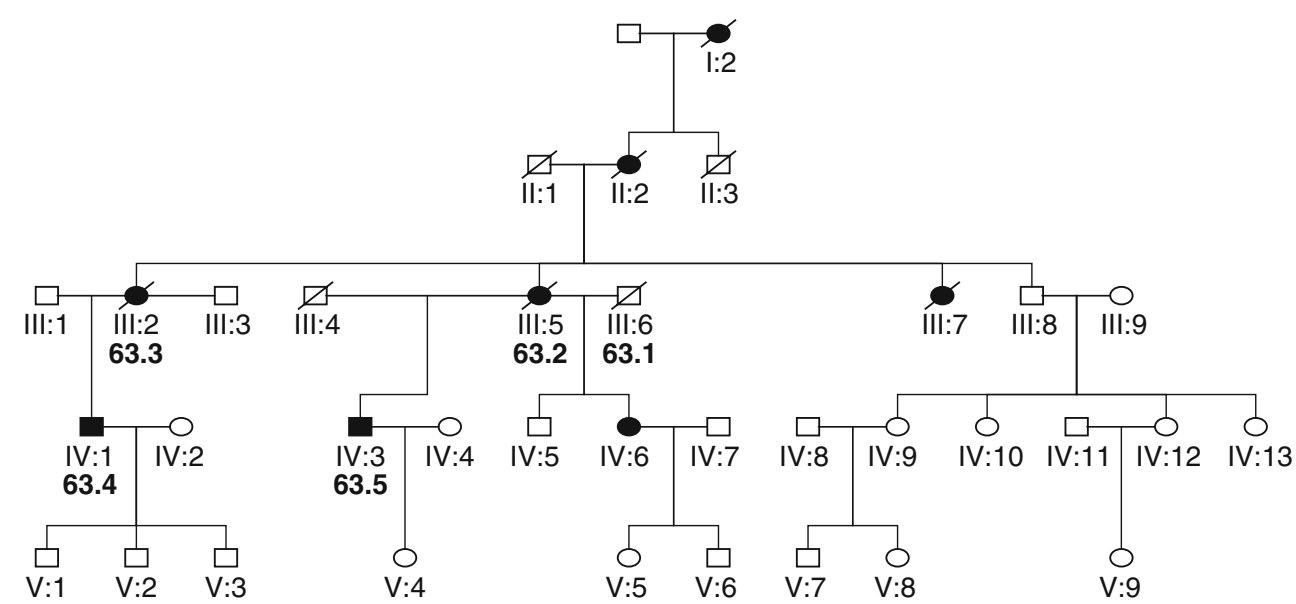


individuals in the family is presented in Table 1. Patients with the Thr17Met mutation appear to benefit from treatment using oral vitamin A supplementation. Individual 63.4 (IV: 1) has been using vitamin A supplementation for 7 years, which has reportedly slowed the progression of his disease (personal communication).

This family was informed that individual molecular testing is now available; however, one affected individual, 63.5 (IV: 3), has refused testing and none of the younger generation $(\mathrm{V})$ has shown interest in receiving molecular results or genetic counseling.

\section{Discussion}

Patients with the Thr17Met mutation generally have a good prognosis: with a relatively later onset and milder phenotype of type 2 adRP [6, 7]. Thr17Met causes an abnormally folded rhodopsin protein that accumulates in the endoplasmic reticulum (ER) of the photoreceptor cell $[7,8]$. The accumulation of misfolded protein in the cell results in impairment of the ubiquitin-proteosome system, which is vital for cellular regulation, and this leads to cytotoxicity and apoptosis $[8,9]$. A potential therapy for patients with this type of misfolding mutation is the use of retinoids as chaperones to improve folding of the protein [9].

In transgenic mice with the Thr17Met mutation, vitamin A supplementation was shown to increase the availability of 11-cis retinal in the retina, binding of which increased the stability of mutant rhodopsin protein, allowed correct localization of the protein and slowed the rate of visual decline [10]. Oral vitamin A supplementation in patients with RP has also been shown to reduce the rate of retinal degeneration [11]. Close medical supervision is required when using Vitamin A supplementation, however, as excessive intake can potentially cause liver toxicity as well as reduced bone density [12]. In addition, Vitamin A has been shown to cause teratogenic effects during pregnancy and may interact with anticonvulsant agents [13].

Patients participating in the RDD research project at the University of Cape Town are motivated by the hope of a cure for themselves or their family members [14]. The identification of an RDD mutation in a family generally means that a molecular diagnosis can be given for members of that family. Patients report that this causes no significant improvement to their day-to-day living. In the case of Thr17Met, mutation carriers can also be given a more accurate disease prognosis, and offered possible therapeutic intervention. Of the 1181 families currently participating in the research program, this is the only case where disease intervention is immediately possible. This particular family can be offered the benefit of molecular-based management and disease intervention; however, affected individual 63.5 (IV: 3) has refused testing and none of the younger generation has made use of the diagnostic service available.

This case highlights a concern regarding the responsibility of the researcher/counselor: should individual 63.5 (IV: 3) and the younger members of the family be actively pursued or should the onus be on them to initiate molecular testing? It has been suggested that an ethical duty to return individual genetic results exists, and that the right of an individual not to receive information can be overridden when there is evidence that the information may benefit others [15]. Vitamin A supplementation may indeed prove beneficial to the mutation positive individuals in the youngest generation of this family (including the daughter of individual 63.5). In this case, the right of individual 63.5 (IV: 3) not to receive his molecular results may be less important than the benefit from therapeutic intervention with Vitamin A supplementation in the younger generation. Adapting the established protocol for the translation of RDD research results, and contacting these individuals may therefore be justifiable.

It has been suggested that genetic testing for RDDs may be associated with a psychological benefit; the fact that "some-
Table 1 Clinical information of individuals from the family with Thr17Met in rhodopsin

\begin{tabular}{|c|c|c|c|c|}
\hline Individual & Mutation & Current Age & Age of Onset & $\begin{array}{l}\text { Other Clinical } \\
\text { Information }\end{array}$ \\
\hline 63.1 & None & Deceased & & \\
\hline 63.2 & $\begin{array}{l}\text { Heterozygous } \\
\text { RHO Thr17Met }\end{array}$ & Deceased & & \\
\hline 63.3 & Not tested & Deceased & 16 & $\begin{array}{l}\text { Nightblindness noticed when young, } \\
\text { subsequent central vision loss, very } \\
\text { poor vision at age } 77 \text {. }\end{array}$ \\
\hline 63.4 & $\begin{array}{l}\text { Heterozygous } \\
\text { RHO Thr17Met }\end{array}$ & 66 & 20 & $\begin{array}{l}\text { Diffuse RP, night blindness, restricted } \\
\text { visual field and myopia, loss of } \\
\text { central vision in left eye at age } 64 \text {. } \\
\text { Vitamin A supplementation from } \\
\text { 1999-2006, vision reported to be } \\
\text { stable during this time }\end{array}$ \\
\hline 63.5 & Refused testing & 64 & & \\
\hline
\end{tabular}


thing is being done" fosters hope and optimism on the part of the patient [16]. In this case, unlike for the majority of RDDs, something can actually be done to decelerate the progression of disease in members of this family. The case presented here serves to highlight the challenges faced by researchers, as well as the benefits provided to the patients, when translational research is undertaken.

Acknowledgments We are indebted to all RDD family members in South Africa for their participation in this research program. This study was funded by Retina South Africa, the University of Cape Town, the South African Medical Research Council, and the Technology and Human Resources for Industry Programme (THRIP) (South Africa).

\section{References}

1. Daiger SP, Bowne SJ, Sullivan LS. Perspective on genes and mutations causing retinitis pigmentosa. Arch Ophthalmol. 2007;125(2):151-8.

2. Hims MM, Diager SP, Inglehearn CF. Retinitis pigmentosa: genes, proteins and prospects. Dev Ophthalmol. 2003;37:109-25.

3. Inglehearn CF, Keen TJ, Bashir R, Jay M, Fitzke F, Bird AC, et al. A completed screen for mutations of the rhodopsin gene in a panel of patients with autosomal dominant retinitis pigmentosa. Hum Mol Genet. 1992;1(1):41-5.

4. Downs K, Zacks DN, Caruso R, Karoukis AJ, Branham K, Yashar $\mathrm{BM}$, et al. Molecular testing for hereditary retinal disease as part of clinical care. Arch Ophthalmol. 2007;125(2):252-8.

5. Greenberg J, Roberts L, Ramesar R. Unusual frequencies of Rhodopsin mutations and polymorphisms in South African patients with Retinitis Pigmentosa. In: Anderson RE, LaVail MM, Hollyfield JG (eds) New insights into retinal degenerative diseases. Proceedings of the 9th International Symposium on
Retinal Degeneration. Durango, CO: Kluwer Academic/Plenum, 2000. pp 329-33.

6. Hayakawa M, Hotta Y, Imai Y, Fujiki K, Nakamura A, Yanashima $\mathrm{K}$, et al. Clinical features of autosomal dominant retinitis pigmentosa with rhodopsin gene codon 17 mutation and retinal neovascularization in a Japanese patient. Am J Ophthalmol. 1993;115(2):168-73.

7. Bell C, Converse CA, Hammer HM, Osborne A, Haites NE. Rhodopsin mutations in a Scottish retinitis pigmentosa population, including a novel splice site mutation in intron four. $\mathrm{Br} \mathrm{J}$ Ophthalmol. 1994;78(12):933-8.

8. Chapple JP, Grayson C, Hardcastle AJ, Saliba RS, van der Spuy J, Cheetham ME. Unfolding retinal dystrophies: a role for molecular chaperones? Trends Mol Med. 2001;7(9):414-21.

9. Mendes HF, van der Spuy J, Chapple JP, Cheetham ME. Mechanisms of cell death in rhodopsin retinitis pigmentosa: implications for therapy. Trends Mol Med. 2005;11(4):177-85.

10. Li T, Sandberg MA, Pawlyk BS, Rosner B, Hayes KC, Dryja TP, et al. Effect of vitamin A supplementation on rhodopsin mutants threonine-17 $\rightarrow$ methionine and proline-347 $\rightarrow$ serine in transgenic mice and in cell cultures. Proc Natl Acad Sci U S A 1998;95(20):11933-8.

11. Berson EL, Rosner B, Sandberg MA, Hayes KC, Nicholson BW, Weigel-DiFranco C, et al. A randomized trial of vitamin A and vitamin E supplementation for retinitis pigmentosa. Arch Ophthalmol. 1993;111(6):761-72.

12. Bartlett H, Eperjesi F. An ideal ocular nutritional supplement? Ophthalmic Physiol Opt. 2004;24(4):339-49.

13. Bartlett H, Eperjesi F. Possible contraindications and adverse reactions associated with the use of ocular nutritional supplements. Ophthalmic Physiol Opt. 2005;25(3):179-94.

14. Basson F, Futter MJ, Greenberg J. Qualitative research methodology in the exploration of patients' perceptions of participating in a genetic research program. Ophthalmic Genet. 2007;28(3):143-9.

15. Knoppers BM, Joly Y, Simard J, Durocher F. The emergence of an ethical duty to disclose genetic research results: international perspectives. Eur J Hum Genet. 2006;

16. Stone EM. Genetic testing for inherited eye disease. Arch Ophthalmol. 2007;125(2):205-12. 